\title{
Le niveau du Saint-Laurent de 2000 BP et l'occupation amérindienne préhistorique de la place Royale à Québec
}

The St. Lawrence River Level of $2000 \mathrm{BP}$ and the Indian Prehistoric Occupation of the Place Royale in Québec City

Der Wasserspiegel des Sankt-Lorenz-Stroms urn 2000 Jahre v.u.Z. und die indianische prähistorische Besiedlung der Place Royale in Québec-Stadt.

\author{
Michel Allard et Jocelyne Séguin
}

Volume 46, numéro 2, 1992

URI : https://id.erudit.org/iderudit/032902ar

DOI : https://doi.org/10.7202/032902ar

\section{Aller au sommaire du numéro}

\section{Éditeur(s)}

Les Presses de l'Université de Montréal

\section{ISSN}

0705-7199 (imprimé)

1492-143X (numérique)

\section{Découvrir la revue}

\section{Citer cet article}

Allard, M. \& Séguin, J. (1992). Le niveau du Saint-Laurent de 2000 BP et l'occupation amérindienne préhistorique de la place Royale à Québec. Géographie physique et Quaternaire, 46(2), 181-188. https://doi.org/10.7202/032902ar

\section{Résumé de l'article}

L'étude d'une coupe de sédiments littoraux à l'occasion de fouilles archéologiques à l'emplacement même des premiers établissements français dans la ville de Québec montre que les Amérindiens ont campé périodiquement sur le site au moins depuis 2000 ans BP. Les analyses stratigraphiques, sédimentologiques et les datations $\mathrm{au}^{14} \mathrm{C}$ sur charbons de bois indiquent qu'à cette date (datations au ${ }^{14} \mathrm{C}$ de $2120 \pm 120,1990 \pm 60$ et de $1970 \pm 80 \mathrm{BP}$ ) les Amérindiens campaient sur le haut de la plage, immédiatement au-dessus du niveau des grandes marées mais sur un site occasionnellement atteint par les crues de tempêtes. En appliquant à ce contexte topographique les paramètres marégraphiques actuels, on calcule que le niveau moyen du Saint-Laurent était à cette époque entre $3,7 \mathrm{~m}$ et 4,2 $\mathrm{m}$ plus haut que présentement. La datation sûre des vestiges archéologiques et la possibilité d'estimer précisément l'altitude du niveau moyen de la mer à cette époque permettent une résolution rarement atteinte dans la détermination des anciens niveaux marins avec les repères géomorphologiques habituellement utilisés et les spécimens généralement datés, tels que les coquillages et le bois flotté. Les résultats obtenus concordent bien avec les données les plus récentes sur les variations du niveau marin relatif dans l'estuaire du Saint-Laurent. 


\section{LE NIVEAU DU SAINT-LAURENT DE 2000 BP ET L'OCCUPATION AMÉRINDIENNE PRÉHISTORIQUE DE LA PLACE ROYALE, À QUÉBEC}

Michel ALLARD et Jocelyne SÉGUIN, Département de géographie et Centre d'études nordiques, Université Laval, Sainte-Foy, Québec G1K 7P4, et Cérane Inc., 975, avenue de Salaberry, Québec, Québec G1R 2V4.

RÉSUMÉ L'étude d'une coupe de sédiments littoraux à l'occasion de fouilles archèologiques à l'emplacement même des premiers établissements français dans la ville de Québec montre que les Amérindiens ont campé périodiquement sur le site au moins depuis 2000 ans BP. Les analyses stratigraphiques, sédimentologiques et les datations au ${ }^{14} \mathrm{C}$ sur charbons de bois indiquent qu'à cette date (datations au ${ }^{14} \mathrm{C}$ de $2120 \pm 120$, $1990 \pm 60$ et de $1970 \pm 80$ BP) les Amérindiens campaient sur le haut de la plage, immédiatement au-dessus du niveau des grandes marées mais sur un site occasionnellement atteint par les crues de tempêtes. En appliquant à ce contexte topographique les paramètres marégraphiques actuels, on calcule que le niveau moyen du Saint-Laurent étaít à cette époque entre $3,7 \mathrm{~m}$ et $4,2 \mathrm{~m}$ plus haut que présentement. La datation sûre des vestiges archéologiques et la possibilité d'estimer précisément l'altitude du niveau moyen de la mer à cette époque permettent une résolution rarement atteinte dans la détermination des anciens niveaux marins avec les repères géomorphologiques habituellement utilisés et les spécimens généralement datés, tels que les coquillages et le bois flotté. Les résultats obtenus concordent bien avec les données les plus récentes sur les variations du niveau marin relatif dans l'estuaire du Saint-Laurent.
ABSTRACT The St. Lawrence River level of $2000 \mathrm{BP}$ and the Indian prehistoric occupation of the Place Royale in Québec City. Interpretation of a section in beach sediments for archeological work at the very emplacement of the first French settlement in Québec City shows that the Indians camped periodically on the site at least since 2000 BP. Stratigraphy, sedimentological analysis and radiocarbon dates on charcoals from camp fires indicate that the natives used at that time $\left({ }^{14} \mathrm{C}\right.$ dates of $2120 \pm 120,1990 \pm 60$ and $1970 \pm 80 \mathrm{BP}$ ) to camp on the top of the beach just above the level of large tides but that the site was occasionally reached by storm surges. Applying present-day tidal parameters to those hydrographic conditions made it possible to calculate that the mean level of the St. Lawrence River was at that time between 3,7 and $4,2 \mathrm{~m}$ above the present level. The very good dating capability offered by the archeological remains and the high precision attainable for determining the elevation of the sea level of that time allow a resolution rarely obtained on raised shorelines with the usual geomorphological indicators and dating materials like shells and driftwood. The results obtained at the old Québec prehistoric site agree well with recently published data on relative sea level variations in the St. Lawrence River Estuary.
ZUSAMMENFASSUNG Der Wasserspiegel des Sankt-Lorenz-Stroms um 2000 Jahre v.u.Z. und die indianische prähistorische Besiedlung der Place Royale in QuébecStadt. Bei archäologischen Ausgrabungen an der Stelle der ersten französischen Niederlassungen in der Stadt Québec hat man einen Schnitt von Küstensedimenten untersucht, der zeigt, daß die Indianer mindestens seit 2000 Jahren v.u.Z. periodisch an dieser Stelle kampiert haben. Die stratigraphischen und sedimentologischen Analysen sowie die ${ }^{14} \mathrm{C}$-Datierungen auf Holzkohle zeigen, daß die Indianer zu dieser Zeit $\left({ }^{14} \mathrm{C}\right.$-Datierungen von $2120 \pm 120,1990 \pm 60$ und $1970 \pm$ 80 v.u.Z.) im oberen Teil des Strandes kampierten, direkt über dem Niveau des Hochwassers aber auf einem Platz, der gelegentlich von den Sturmfluten erreicht wurde. Wenn man auf diesen topographischen Kontext die gegenwärtigen Flutparameter anwendet, errechnet man, daß das Durchschnittsniveau des Sankt-Lorenz-Stroms zu jener Zeit zwischen $3,7 \mathrm{~m}$ und $4,2 \mathrm{~m}$ höher war als gegenwärtig. Die zuverlässige Datierung der archäologischen Spuren und die Möglichkeit, die Höhe des durchschnittlichen Meeresniveaus damals genau zu bestimmen, erlauben eine Genauigkeit bei der Bestimmung der ehemaligen Meeresniveaus, die mit den gewöhnlich benutzten geomorphologischen Anhaltspunkten und dem im allgemeinen datierten Material wie Muscheln und Treibholz selten erreicht wird. Die erhaltenen Ergebnisse stimmen mit den neuesten Daten über die Variationen des relativen Meeresspiegels im Mündungsbecken des SanktLorens-Stroms gut überein. 


\section{INTRODUCTION}

Les travaux récents de Dionne (1988 a et b) dans l'estuaire du Saint-Laurent, particulièrement dans la région de Montmagny, ont mis en évidence une succession d'oscillations du niveau relatif de la mer durant l'Holocène. Ces variations furent plus complexes que ne le proposaient auparavant les courbes d'émersion postglaciaire. Parmi les lignes de rivage anciennes, le niveau Mitis est particulièrement bien marqué dans le paysage. En effet, il est associé à la terrasse qui s'élève à quelques mètres au-dessus de la ligne des hautes eaux actuelles. Cette terrasse se serait formée par suite de l'accrétion, entre 3000 et $1200 \mathrm{BP}$, de sédiments littoraux sur une plate-forme d'érosion plus ancienne (Dionne, 1977, 1990).

L'âge et l'altitude d'un niveau marin correspondant à la terrasse Mitis ont pu être estimés de façon précise grâce à l'étude des sédiments littoraux et à la datation des vestiges indiens préhistoriques au site de la place Royale, dans le cœur de la vieille ville de Québec.

\section{LE SITE ARCHÉOLOGIQUE DE LA PLACE ROYALE}

Les coupes étudiées se trouvent à la place Royale dans la basse-ville de Québec, à environ $20 \mathrm{~m}$ du parvis de l'église Notre-Dame-des-Victoires (fig. 1). L'analyse d'une première coupe stratigraphique à cet endroit avait pour but d'interpréter l'origine des lits de sable et gravier disposés sous un horizon d'une dizaine de centimètres d'épaisseur dans lequel abondent des vestiges archéologiques. Cet horizon est associé de toute évidence à l'occupation amérindienne préhistorique et constitue la surface du sol sur lequel se sont établis les premiers colons français sous l'autorité de Samuel de Champlain, en 1608 ap. J.-C. Son altitude est actuellement de 9,1 m au-dessus du niveau de référence géodésique.

Les cartes et les croquis datant du début du régime français, principalement ceux de Bourdon, dans Painchaud (1982) ainsi que la carte géomorphologique de Morneau (1989) montrent qu'à son état naturel, le site occupait une terrasse marine à quelques mètres au-dessus du niveau des grandes marées. Cette terrasse est constituée de sable très grossier, de cailloux et de galets de schiste. De faible étendue, elle occupait, au pied du cap Diamant, une superficie de l'ordre de $25000 \mathrm{~m}^{2}$ avant les remblayages qui accompagnèrent l'expansion urbaine. Ces dépôts d'origine littorale constituent, comme l'indiquent les sondages compilés par Painchaud, une couverture meuble de quelques mètres d'épaisseur $(1,7 \mathrm{~m}$ au droit du lieu de fouilles) reposant sur une plate-forme rocheuse au relief inégal. La plate-forme rocheuse affleurait au bas de la plage, au niveau des basses mers (fig. 1). Si on fait abstraction du paysage urbain, il apparaît que le site offrait de bonnes possibilités comme aire d'occupation et de transit: accès au sommet de la falaise par un sentier (à l'emplacement de l'actuelle Côte de la Montagne); abordage facile en embarcation grâce à une plage qui, compte tenu du grand marnage et de sa nature sableuse et caillouteuse, devait avoir une pente assez forte (donc pas de vasière à traverser à marée basse); potentiel de pêche sur les estrans environnants, notamment à l'embouchure de la rivière Saint-Charles; vue sur le fleuve et la circulation des canots; abri au pied de la falaise contre les vents du sud-ouest, dominants dans la vallée du Saint-Laurent. Toutefois, la superficie disponible pour les campements au pied du cap Diamant était plutôt restreinte et les terrains bas de la rivière Saint-Charles offraient plus d'espaces et de ressources. C'est d'ailleurs dans les environs que se trouvait probablement le village de Stadacone, comme l'a noté Jacques Cartier en 1534 et 1535 , lors de ses visites.

Au site de la place Royale, la collection d'objets amérindiens en céramique est composée "de vases, de pipes et de perles qui se classent dans les traditions du Sylvicole Moyen ancien (400 BC - $500 \mathrm{AD}$ ) et du Sylvicole Moyen tardif $1500-$ 1000 AD). Pendant le temps du Sylvicole Moyen ancien, la pointe de Québec devait surtout être un lieu de transit de petits groupes régionaux venus passer quelques jours à une halte routière. On dressait alors des camps temporaires, on y taillait une bonne provision d'outils et on vaquait aux affaires courantes" (Cérane Inc., 1989, p. VII).

\section{MÉTHODES}

Les horizons étudiés se situent sous quelque 1,5 m de remblais de la période historique débutant avec l'implantation européenne.

L'observation des couches de sables et graviers sousjacents aux horizons archéologiques et leur interdigitation occasionnelle avec ceux-ci a été faite en juin 1988, à l'occasion des travaux archéologiques. L'interprétation et l'échantillonnage d'une coupe particulière (fig. 2a) à une étape donnée lors des travaux d'excavation a permis de reconnaître le caractère littoral de ces sédiments et de les décrire. Subséquemment, une autre coupe (fig. 2b) ainsi que l'analyse du nivellement géodésique précis ont permis de tirer des interprétations sur l'emplacement en haut de plage des premiers vestiges anthropiques. Les datations au radiocarbone de ces vestiges, principalement celles réalisées sur des charbons de bois, ont fourni l'âge précis de l'époque où les Amérindiens ont exercé des activités en marge des plus hautes marées.

Le tamisage pour les analyses granulométriques des sables et graviers a été limité à 12 minutes, compte tenu de la fragilité des plaquettes schisteuses qu'ils contiennent. Afin d'évaluer le degré d'évolution des sédiments de plage, l'émoussé $R_{1}$ et $R_{2}$ (Cailleux et Tricart, 1959) de cent cailloux compris entre 2,5 et $8,5 \mathrm{~cm}$ de longueur de chaque couche minérale a été mesuré. Pour des fins de comparaison, cent cailloux d'un échantillon prélevé dans un éboulis naturel au pied de la falaise à peu de distance ont aussi été mesurés. Les schistes, largement dominants dans le dépôt, ne se prêtent guère à ce genre d'analyse car, plaquettes fragiles, ils peuvent casser au brassage et toujours présenter des arêtes aiguës; ça n'empêche toutefois pas le développement d'une certaine usure des arêtes par abrasion dans l'eau.

Afin d'évaluer le degré d'évolution chimique des horizons dans la coupe, on a procédé à trois analyses différentes: la mesure du $\mathrm{pH}$, la teneur en carbone (en\%) et la teneur en azote (en\%) (tabl. 1). Les analyses ont été faites selon les méthodes standard de pédologie (Commission canadienne de pédologie, 1978). Les rapports carbone/azote $(C / N)$ permettent une appréciation de l'état de décomposition de la matière organique contenue dans les horizons. 


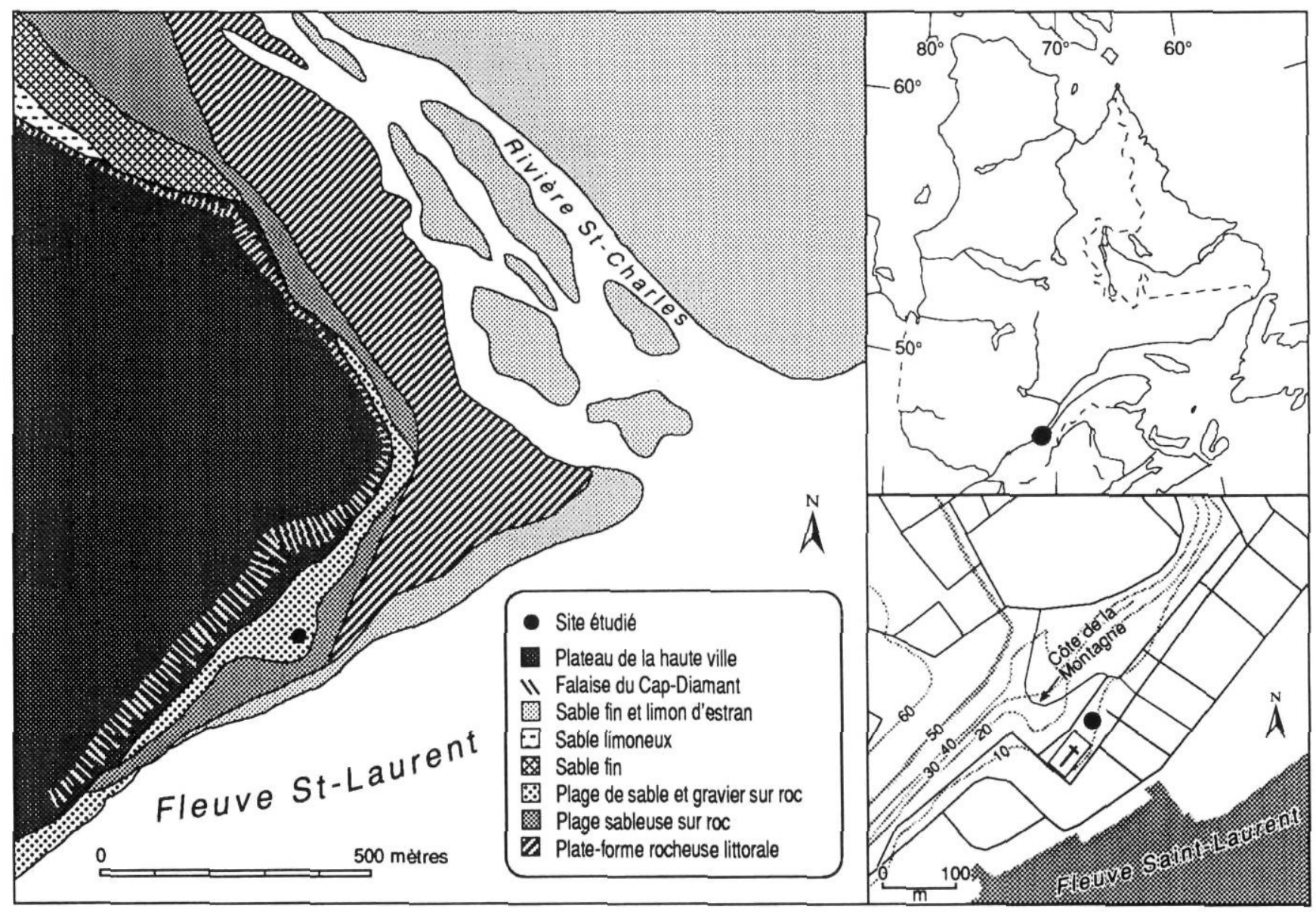

FIGURE 1. Localisation du site de la place Royale dans la ville de Québec et cadre paléo-géomorphologique. Le croquis a été modifié à partir de la carte géomorphologique de Morneau (1989), reconstituée à partir de plans historiques et de données techniques (courbes de niveau en mètres).
Location map of the Place Royale site in Québec City, and original geomorphological context; this sketch was extracted and modified from the geomorphological map of Morneau (1989), reconstructed from historical and technical sources (contours in metres).
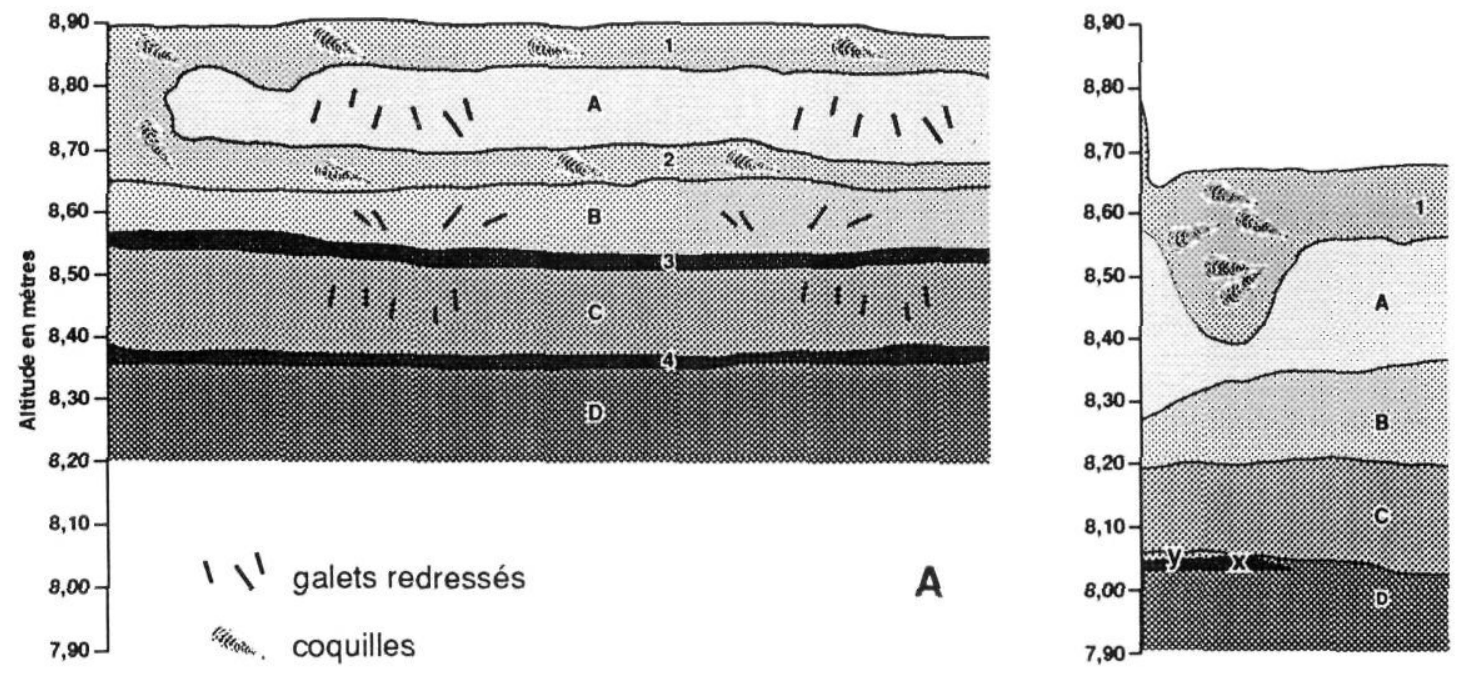

FIGURE 2. A) Croquis de la coupe étudiée. B) Croquis d'une coupe voisine à $6,4 \mathrm{~m}$ de distance. Le $\mathrm{X}$ indique le lit de charbon de bois de $2120 \mathrm{BP}$ et le $\mathrm{Y}$, la disposition des galets rougis au feu associés. Le sommet de la couche 1 était tronqué par un remplissage d'âge historique.
A) Sketch of the studied section. B) Sketch of a neighbouring section 6,4 $m$ apart. The $X$ indicates the charcoal bed dated at $2120 \mathrm{BP}$ and the $Y$ shows the bed of associated burnt coloured pebbles. The top part of layer 1 was truncated by a filling of historical age. 
TABLEAU ।

Analyses chimiques des horizons de la coupe étudiée

\begin{tabular}{ccccc}
\hline couche & $\mathrm{pH}$ & $\% \mathrm{C}$ & $\% \mathrm{~N}$ & $\mathrm{C} / \mathrm{N}$ \\
\hline $1(0-5 \mathrm{~cm})$ & 7,40 & 3,65 & 0,46 & 7,93 \\
$1(5-10 \mathrm{~cm})$ & 7,50 & 3,65 & 0,42 & 8,69 \\
A & 7,60 & 2,09 & 0,32 & 6,53 \\
2 & 7,60 & 4,23 & 0,44 & 9,61 \\
B & 8,30 & 0,52 & 0,14 & 3,71 \\
3 & 8,30 & 0,81 & 0,18 & 4,50 \\
C & 8,30 & 1,04 & 0,20 & 5,20 \\
4 & 8,30 & 0,52 & 0,16 & 3,25 \\
D & 8,30 & 0,46 & 0,16 & 2,87 \\
\end{tabular}

En tout huit datations au carbone 14 ont été effectuées sur des charbons de bois, trois sur des ossements humains, une sur des coquilles et une sur de la matière carbonisée associée à un vase (tabl. II).

\section{LA COUPE ÉTUDIÉE}

Située au fond du chantier de fouilles, la coupe étudiée en mai 1988 fait $86 \mathrm{~cm}$ de hauteur (de $8,24 \mathrm{~m}$ à 9,1 $\mathrm{m}$ d'altitude) et $1,1 \mathrm{~m}$ de front. Grâce à l'excavation systématique assurée par l'équipe d'archéologues, elle était visible sur trois faces. À l'endroit précis où elle a été levée, la coupe comprenait quatre couches organiques séparées par autant de couches de sables et de cailloux (fig. 2 a et 3 ). Aux alentours, cette stratigraphie est variable, les couches organiques se rejoignant latéralement à la faveur de l'amincissement ou de l'interruption des couches sablo-graveleuses pour ne constituer qu'une seule couche organique qui atteint localement $60 \mathrm{~cm}$ d'épaisseur. Ici et là également, les galets schisteux des horizons minéraux, généralement déposés à plat, ont été perturbés par l'activité humaine (brassage, trous de piquets, etc.). Les couches organiques, particulièrement celle du dessus, renferment de nombreuses coquilles d'Elliptio complanatus, une huître d'eau douce comestible qui devait abonder sur les estrans avoisinants. À l'exception d'une proportion minime d'éléments granitiques, de calcaires des basses terres et de cailloux appalachiens allochtones au site (probablement des apports glaciels), les cailloux des couches minérales sont d'origine locale et consistent en schistes argileux et en plaquettes microgréseuses de la formation de Québec.

La couche 1, riche en vestiges anthropiques, fait un peu plus de $10 \mathrm{~cm}$ d'épaisseur (fig. 2a). La matière organique noire est disséminée à travers les cailloux schisteux. Malgré la teinte foncée du matériel, la teneur en carbone organique n'est que de $3,65 \%$ (tabl. I). Les âges au ${ }^{14} \mathrm{C}$ obtenus sur le chantier de fouilles dans cette couche continue sur des charbons, des restes de nourriture (matière organique carbonisée collée à un vase, coquilles vides) et des ossements humains s'étalent de $190 \pm 70$ jusqu'à $1970 \pm 80 \mathrm{BP}$ (tabl. II). On peut en conclure que depuis $1970 \mathrm{BP}$ le site est resté émergé et hors de portée des crues de tempêtes.

La couche $A$, juste en dessous est composé du même genre de cailloutis. Au centre de la coupe, les cailloux sont
TABLEAU ॥

Datations au ${ }^{14} \mathrm{C}$ du site de la place Royale

\begin{tabular}{|c|c|c|c|}
\hline $\mathrm{N}^{\circ}$ d'échantillon & Date BP & Type & $\begin{array}{l}\text { Altitude au-dessus } \\
\text { du niveau moyen } \\
\text { actuel de la mer }\end{array}$ \\
\hline Beta-28460 & $190 \pm 70$ & $\begin{array}{l}\text { charbon de } \\
\text { foyer }\end{array}$ & $8,72-8,76$ \\
\hline Beta-28457 & $210 \pm 100$ & $\begin{array}{l}\text { charbon de } \\
\text { foyer }\end{array}$ & $9,12-9,24$ \\
\hline $\begin{array}{c}\text { Beta-26468; ETH- } \\
4271\end{array}$ & $715 \pm 70$ & $\begin{array}{l}\text { ossements de } \\
\text { sépulture }\end{array}$ & $8,80-9,12$ \\
\hline RL-1831 & $790 \pm 100$ & $\begin{array}{l}\text { ossements de } \\
\text { sépulture }\end{array}$ & $8,80-9,12$ \\
\hline Beta-28461 & $830 \pm 60$ & $\begin{array}{l}\text { charbon de } \\
\text { foyer }\end{array}$ & $8,63-8,72$ \\
\hline $\begin{array}{c}\text { Beta-26467; ETH- } \\
4270\end{array}$ & $930 \pm 65$ & $\begin{array}{l}\text { ossements de } \\
\text { sépulture }\end{array}$ & $8,80-9,12$ \\
\hline $\begin{array}{r}\text { Beta-26469; } \\
\text { ETH-4272 }\end{array}$ & $1175 \pm 75$ & carbonisation & $8,80-9,12$ \\
\hline UL-570 & $1360 \pm 60$ & $\begin{array}{l}\text { coquilles } \\
\text { (restes } \\
\text { alimentaires) }\end{array}$ & $8,80-8,90$ \\
\hline Beta-28458 & $1640 \pm 130$ & $\begin{array}{l}\text { charbon de } \\
\text { foyer }\end{array}$ & $8,92-9,01$ \\
\hline Beta-28463 & $1670 \pm 60$ & $\begin{array}{l}\text { charbon de } \\
\text { foyer }\end{array}$ & $8,40-8,50$ \\
\hline UL-575 & $1970 \pm 80$ & $\begin{array}{l}\text { charbon d'une } \\
\text { dépression }\end{array}$ & $8,78-9,06$ \\
\hline Beta-28462 & $1990 \pm 60$ & $\begin{array}{l}\text { charbon de } \\
\text { foyer }\end{array}$ & $8,39-8,34$ \\
\hline Beta-28459 & $2120 \pm 120$ & $\begin{array}{l}\text { charbon de } \\
\text { foyer }\end{array}$ & $8,05-8,08$ \\
\hline
\end{tabular}

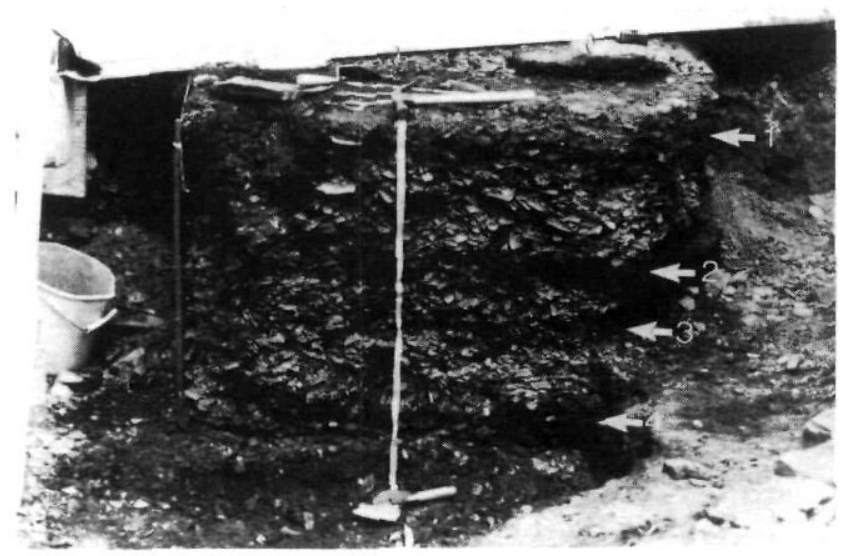

FIGURE 3. La coupe à l'étude, fin mai 1988; les flèches indiquent les quatre couches organiques.

The studied section, end of May 1988; arrows indicate the four organic layers. 
légèrement redressés, probablement à cause d'une perturbation de nature anthropique. Les histogrammes d'émoussés $R_{1}$ et $R_{2}$ (fig. $4 c$ et d) montrent, en comparaison avec les cailloux d'éboulis (fig. 4a et b), un émoussé acquis par usure dans l'eau. Malgré l'absence sur le terrain d'une coloration foncée bien évidente, la teneur en carbone organique atteint 2,09\%, une valeur supérieure à celle des autres horizons minéraux, voire même des couches organiques 3 et 4 . Cette teneur relativement élevée résulte selon toute probabilité d'une infiltration après le dépôt de la couche 1.

La couche 2 se compare à la première quant au faciès et au contenu en restes anthropiques. Cependant, elle est plus mince (environ $5 \mathrm{~cm}$ ) et pauvre en coquilles. Sa teneur en carbone organique est de 4,23\%. Plus en profondeur, les couches organiques 3 et 4 sont plus minces, pauvres en carbone organique et ne contiennent pas de restes anthropiques, ce qui indique qu'elles représentent probablement des phases initiales de stabilisation pédologique.

Les couches minérales $B$ et $D$ contiennent un peu plus de sable que les couches $A$ et $C$ (fig. 5). L'émoussé des cailloux augmente avec la profondeur (fig. 4). La couche D appartient aux sédiments littoraux non remaniés. En effet, aucun reste archéologique ni indice de perturbation anthropique éventuelle n'y apparaît. L'excavation fut suffisamment profonde pour
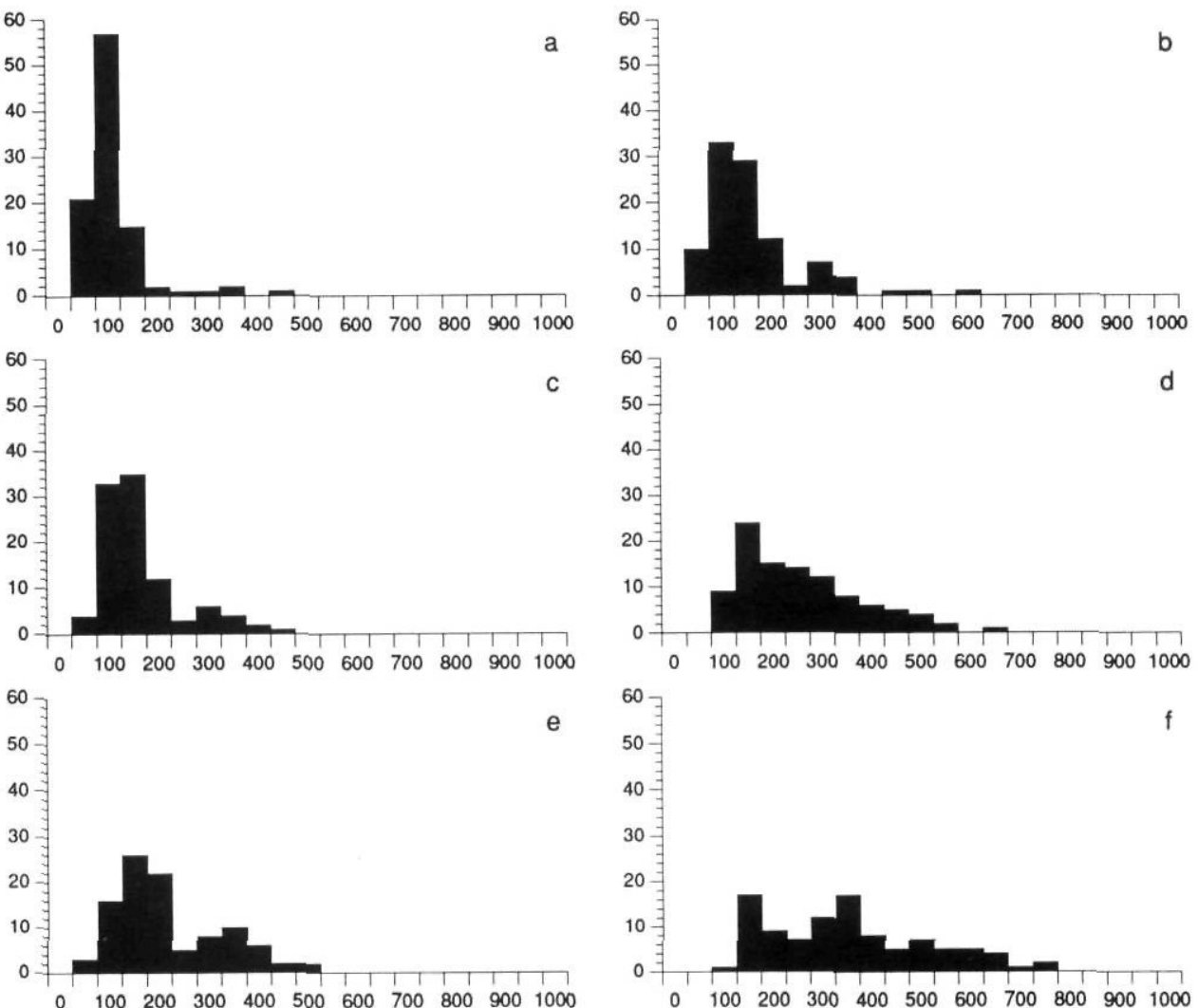

FIGURE 4. Histogrammes des indices d'émoussés (Cailleux et Tricart, 1959): a) émoussés R1 des cailloux de l'éboulis actuel; b) émoussés R2 de l'éboulis actuel; c) émoussés R1 du niveau $A$; d) émoussés R2 du niveau $A$; e) émoussés R1 du niveau B; f) émoussés $R 2$ du niveau $B$; g) émoussés R1 du niveau $C$; h) émoussés R2 du niveau C; i) émoussés $R 1$ du niveau $D$; j) émoussés R2 du niveau $D$.

Histograms of roundness indices (Cailleux and Tricart, 1959); a) R1 roundness indices of pebbles from present scree; b) $R 2$ indices from present scree; c) $R 1$ indices from layer $A$; d) $R 2$ indices from layer $A$; e) $R 1$ indices from layer $B$; f) $R 2$ indices from layer $B ; g) R 1$ indices from layer $C$; h) $R 2$ indices from layer $C$; i) $R 1$ indices from layer $D$; j) $R 2$ indices from layer $D$.
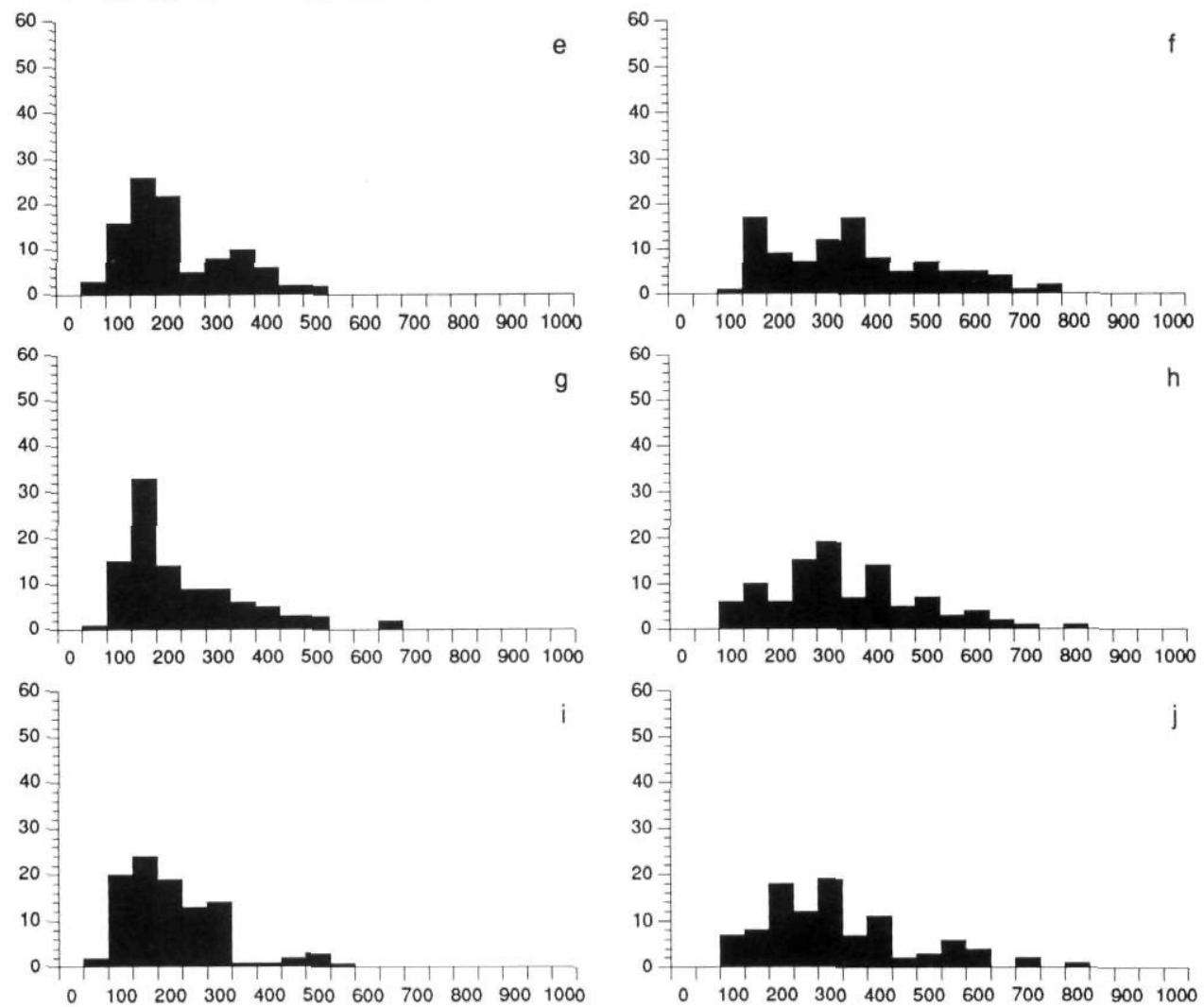
savoir qu'il n'y a pas d'autres horizons organiques intercalés plus bas. Ces sédiments consistent en un sable très grossier jaune-rouge avec de petits granules émoussés de schistes rouges et gris. Les galets y sont disposés à plat. Relativement à celui des autres niveaux, le matériel est particulièrement émoussé (fig. $4 \mathrm{i}$ et j). La teneur en carbone organique est la plus faible de toutes $(0,46 \%)$, indiquant ainsi que le sédiment n'a subi aucune pédogenèse. Son faciès est en tous points comparable à celui des plages schisteuses anciennes et actuelles de l'estuaire du Saint-Laurent.

Les $\mathrm{pH}$ des couches $1, A$ et 2 , soit les trois premières du dessus, sont de l'ordre de 7,5 à 7,6, tandis que celui des couches inférieures est de 8,3. Ceci s'explique par une humi- fication des horizons les plus riches en matière organique (tabl. I) tandis que les niveaux $\mathrm{B}, 3, \mathrm{C}, 4$ et $\mathrm{D}$ ont des $\mathrm{pH}$ qui reflètent simplement la nature légèrement cabonatée des schistes. Les rapports $\mathrm{C} / \mathrm{N}$, tous inférieurs à 10 , révèlent un taux de décomposition avancée de la matière organique en raison de l'activité biologique post-sédimentaire.

\section{AUTRES DONNÉES DU CHANTIER DE FOUILLES}

Les couches sablo-graveleuses et les couches organiques discontinues s'inclinent légèrement vers le nord-est, soit vers le fleuve. Ainsi, à environ 6,4 m de la première coupe, une autre face du chantier montrait une stratigraphie apparentée mais différente (fig. $2 b$ ). Sous l'horizon organique épais de la couche

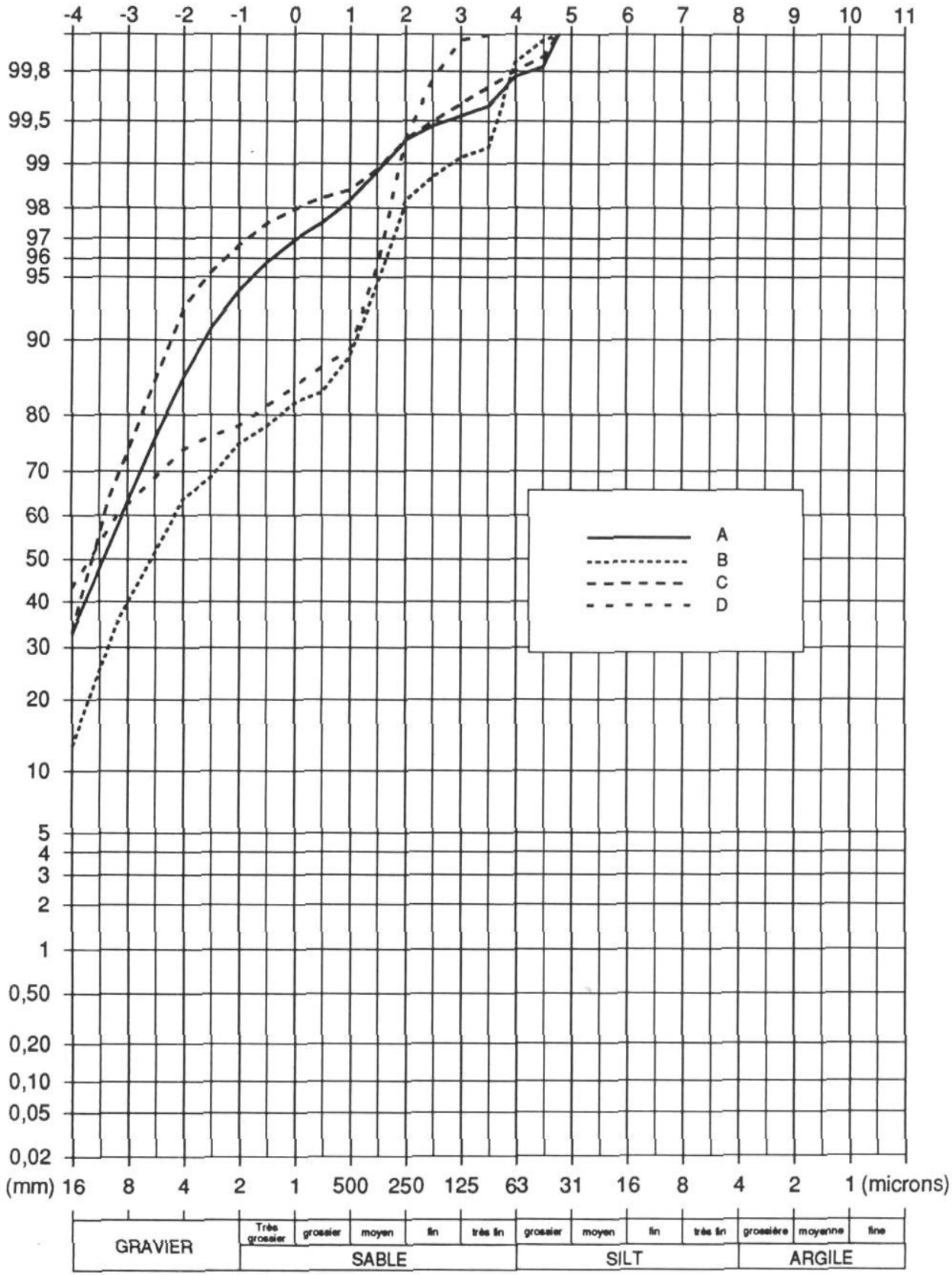

ÉCHELLE DE WENTWORTH MODIFIÉE
FIGURE 5. Courbes granulométriques des sédiments des couches A,B,C,D.

Grain size distribution curves of layers $A$ to $D$. 
1, continue sur le chantier, s'étage une séquence de lits de cailloux comparable en texture et en émoussé aux couches A, B, $C$ et $D$ mais sans horizons organiques intercalés. Toutefois des charbons de bois sont présents dans le niveau $\mathrm{D}$, à 8,05 m d'altitude. Des galets rougis au feu se trouvaient juste au-dessus des charbons. À la limite du chantier de fouilles, la structure n'a pas été excavée en totalité et aucun artefact n'y était associé. La présence des galets rougis témoigne fort probablement d'un feu assez intense pour altérer les cailloux. L'âge des charbons de bois est de $2120 \pm 120 \mathrm{BP}$, soit le plus vieux du site.

\section{LE NIVEAU MARIN D'IL Y A 2000 ANS}

Le fait qu'aucune pédogénèse ne soit apparente dans la couche D laisse croire fortement que l'activité humaine associée à la couche 4 s'est faite sur la plage vive, c'est-à-dire sur un substrat sans couvert végétal et encore à la portée occasionnelle des crues de tempête lesquelles surviennent généralement tard l'automne à Québec. Cette déduction est en accord avec les conclusions des recherches archéologiques à l'effet que le site n'était utilisé que l'été par les Amérindiens (Cérane Inc., 1989). De cette déduction, il découle que le niveau moyen du fleuve devait être plus haut à cette époque qu'aujourd'hui. De combien? On peut formuler un calcul fondé sur les conditions marégraphiques actuelles. Ainsi, selon les tables de marées (Canada, 1990), le niveau extrême de pleine mer à Québec est de 7,1 m au-dessus du zéro marégraphique et le niveau moyen de l'eau est à 2,6 $\mathrm{m}$ au-dessus de ce même zéro de référence. Ceci implique que les niveaux extrêmes, lors de tempêtes, atteignent une hauteur de 4,5 m (7,1 m 2,6 m) au-dessus du niveau moyen de la mer. Or, les valeurs de nivellement topographique terrestre utilisées dans l'arpentage du site ont comme référence le zéro géodésique qui correspond à quelques centimètres près au niveau moyen actuel. Si l'on tient pour acquis que les conditions océanographiques et climatiques n'ont guère dû changer, le niveau moyen de l'eau se trouvait donc à environ 6,34 m (tabl. III) par rapport au zéro géodésique actuel, soit 3,74 m plus haut qu'aujourd'hui lorsque les premiers utilisateurs ont pu occuper le haut de la plage (couche 4). Par corrélation stratigraphique, on peut associer à ce niveau de $8,24 \mathrm{~m}$ les charbons de bois datés de $2120 \pm 120$ BP et situés à $8,05 \mathrm{~m}$. Vu son contexte (fig. $2 \mathrm{~b}$ ), ce feu a été fait sur la plage vive alors que quelques mètres plus loin et quelques décimètres plus haut on campait sur le terrain juste au-dessus du niveau des hautes mers.

On observe que vers le haut de la coupe étudiée les couches sédimentaires intercalées aux niveaux d'occupation humaine ont des cailloux de moins en moins émoussés et que l'ampleur des perturbations de la stratigraphie (ondulations, cailloux sur la tranche) augmente. Ceci laisse supposer deux choses: 1) que le remaniement marin est devenu progressivement moins important à cause de l'influence décroissante des tempêtes, associée peut-être à la baisse du niveau marin et 2) que l'occupation humaine s'intensifiait en durée d'une couche à l'autre. Selon une autre hypothèse, vers l'époque de la stabilisation définitive, avant la couche 2 (niveau B) et avant la couche 1 (niveau A), des cailloux de plage et peut-être d'éboulis auraient été redistribués sur le site par les occupants.

\section{TABLEAU III}

Calcul de la différence d'élévation du niveau moyen du fleuve entre le début de l'occupation du site et aujourd'hui

Données de base:

- Altitude de la couche $4=8,24 \mathrm{~m}$ au-dessus du zéro géodésique

- Niveau moyen de l'eau $=0$ géodésique $=2,6 \mathrm{~m}$ au-dessus du zéro marégraphique

- Écart entre le niveau moyen de l'eau et le niveau extrême de l'eau: $7,1 m-2,6 m=4,5 m$

Calcul:

Altitude (géodésique) de la couche $4=$ $8,24 m$

Différence entre 0 géodésique et 0 des marées $=$ $2,60 \mathrm{~m}$

Altitude actuelle du site au-dessus du zéro marégraphique $=$

$10,84 \mathrm{~m}$

Écart entre niveau moyen de l'eau et niveau extrême $=\quad 4,50 \mathrm{~m}$

Altitude géodésique du niveau moyen de l'eau lorsque la couche 4 était le haut de la plage $=$ $6,34 \mathrm{~cm}$

Différence actuelle entre le zéro des marées et le zéro géodésique $=$

$2,60 m$

Différence de hauteur du niveau moyen de la mer entre autrefois $($ couche 4$)$ et maintenant =

$3,74 \mathrm{~m}$

Deux dates comparables à des altitudes un peu plus élevées, soit $1990 \pm 60 \mathrm{BP}$ (ca 8,32 m) et 1970 $\pm 80 \mathrm{BP}$ (ca $8,8 \mathrm{~m})$, appartiennent à des horizons d'occupations plus stables. Si l'interprétation sédimentologique est juste, ces dates correspondent à une période où le site n'était plus qu'exceptionnellement atteint par les crues de tempête. Si on applique le même calcul que précédemment, on arrive à un niveau moyen de la mer de l'ordre de 4,3 m plus haut à cette époque qu'aujourd'hui. II est possible d'argumenter de plusieurs points de détail. Les couches de graviers de moins en moins émoussés intercalés entre les couches d'occupation humaine peuvent refléter des crues de tempêtes espacées dans le temps et être associées à des oscillations du niveau marin relatif. Quelle influence l'activité des Amérindiens a-t-elle pu avoir sur le modelé de détail du haut de plage (nivellement de fond de tentes, piétinement, etc.)? Une seule crue de forte tempête peut facilement redistribuer une tranche de cailloux schisteux de quelques centimètres sur le haut d'une plage. La nature des sédiments en cause et la limite de précision des datations ${ }^{14} \mathrm{C}$ empêchent de pousser plus loin les interprétations. II n'en reste pas moins que les données démontrent que les Amérindiens occupaient le rebord supérieur de la plage, y campaient et y faisaient des feux il y a précisément 2000 ans. Ce haut de plage devait correspondre à un niveau marin entre 3,7 et 4,3 m plus haut qu'aujourd'hui.

\section{CORRÉLATIONS}

L'ampleur de l'abaissement du niveau marin relatif, de l'ordre de 4 m, de 2000 BP à aujourd'hui correspond aux données 
les plus récentes recueillies le long de l'estuaire du SaintLaurent. Dionne (1988a) résume ainsi les variations holocènes du niveau marin relatif dans l'estuaire du Saint-Laurent: après l'émersion postglaciaire rapide, le niveau marin aurait été plus bas qu'actuellement de 7000 à $6000 \mathrm{BP}$, puis une remontée d'une dizaine de mètres se serait produite de 5800 à 4500 BP; vers $3000 \mathrm{BP}$, le niveau marin s'était abaissé à peu près à celui d'aujourd'hui pour remonter encore jusqu'à $4-5 \mathrm{~m}$ et enfin redescendre depuis 2000 ans environ. L'analyse du site de la place Royale corrobore l'élévation du niveau marin à $4 \mathrm{~m} \pm$ $0,3 \mathrm{~m}$ il y a exactement 2000 ans BP.

La datation des charbons de bois de feux de camp ne laisse aucun doute quant à la contemporanéité entre le matériel daté et sa mise en place sur le site, tandis que l'établissement des campements hors de portée de la marée garantit la qualité de la mesure altimétrique du paléoniveau estuarien. Les analyses sédimentologiques, chimiques et les interprétations archéologiques appuient aussi les conclusions quant à la position du site relativement au niveau marin de l'époque. Enfin, la station marégraphique de Québec est heureusement une des rares au pays pour lesquelles le niveau des crues de tempêtes a été calculé, en raison de sa longue durée de fonctionnement. Le niveau et l'âge obtenus sont d'une précision exceptionnelle comparativement à la datation de niveaux marins anciens à l'aide de coquillages, de bois flottés et d'autres débris organiques sur les côtes à grandes marées comme l'estuaire du Saint-Laurent. Le niveau daté correspond très bien à la terrasse Mitis dont l'âge estimé par Dionne (1990), à l'aide d'une vingtaine de datations, se situe entre 3000 et $1200 \mathrm{BP}$.

Les découvertes récentes sur les variations holocènes du niveau marin relatif dans l'estuaire du Saint-Laurent auront aussi des répercussions sur les recherches archéologiques. II est en effet probable que certains sites paléo-indiens et archaïques très anciens soient maintenant submergés et que plusieurs autres, établis avant $2000 \mathrm{BP}$, aient été détruits lors de transgressions.

\section{REMERCIEMENTS}

Les auteurs remercient l'équipe de terrain de Cérane Inc. qui a prélevé soigneusement les échantillons tout en poursuivant les fouilles archéologiques. Les analyses sédimentologiques ont été faites par $M$. Jean Lavoie au laboratoire de
Géographie physique de l'Université Laval et les analyses chimiques par le laboratoire de Chimie des sols de la même institution, sous la direction du Dr Michel Cescas. L'étude archéologique de Cérane Inc. a été commandée et financée par la ville de Québec, division du Vieux-Québec et du patrimoine et par le ministère des Affaires culturelles du gouvernement du Québec dont les chargés de projet étaient M. William Moss d'une part et $\mathrm{M}^{\mathrm{m} \mathrm{e}}$ Louise Décarie et M. Gilles Samson d'autre part. L'analyse des données archéologiques a été réalisée par MM. Norman Clermont et Claude Chapdelaine de l'Université de Montréal. Les commentaires des deux lecteurs critiques de la revue, MM. Pierre Gangloff et Gilbert Prichonnet, ont été fort utiles. M. Pierre J. H. Richard a agi à titre de rédacteur dans l'évaluation du manuscrit. L'interprétation proposée n'engage que les auteurs.

\section{RÉFÉRENCES}

Cailleux, A. et Tricart, J., 1959. Initiation à l'étude des sables et galets. Sedes, Paris, $376 \mathrm{p}$.

Canada, 1990. Table des marées et courants du Canada, Volume 3, Fleuve Saint-Laurent et rivière Saguenay. Pêches et Océans Canada, Service hydrographique, publication annuelle, $39 \mathrm{p}$.

Cérane Inc., 1989. L'occupation historique et préhistorique de la Place Royale. Rapport prèsentẻ au ministère des Affaires culturelles du Québec et à la ville de Québec, 426 p.

Commission canadienne de pédologie, 1978. Le système canadien de classification des sols. Agriculture Canada, Direction de la recherche, Publication $1646,170 \mathrm{p}$.

Dionne, J.-C., 1977. La mer de Goldthwait au Québec. Géographie physique et Quaternaire, 31: 61-80.

-1988a. Holocene relative sea-level fluctuations in the St. Lawrence Estuary, Québec, Canada. Quaternary Research, 29: 233-244.

1988b. L'émersion de la côte sud du Saint-Laurent depuis la dernière glaciation. Geos, 17: 18-21.

1990. Observations sur le niveau marin relatif à l'Holocène, à Rivièredu-Loup, estuaire du Saint-Laurent, Québec. Géographie physique et Quaternaire, 44: 43-53.

Morneau, F., 1989. Contribution à une méthodologie de caractérisation et de cartographie écologique en milieu urbain: le cas de la basse-ville de Québec. Université Laval, Centre de recherches en aménagement et en développement. Les Cahiers du CRAD, 12(4), 113 p.

Painchaud, A., 1982. Paléogéographie du site archéologique CeEt-9, Place Royale, Pointe de Québec. Cérane Inc., 111 p. 